\title{
On the role of fluid characteristics on promoting microbiologically influenced corrosion (MIC)
}

\section{Editorial}

Corrosion in general and microbiologically influenced corrosion (MIC) is among the most important and most significant sources of losing mechanical integrity as well as economic loss and ecological damage. To illustrate how significant MIC is we have to define its impact within the context of corrosion and its impacts:

Take the economic impact of corrosion (and therefore MIC). Studies carried out for more than 70 years in various countries and industry sectors show that the cost of corrosion varies between 1 to $5 \%$ of the Gross Domestic Product (GDP) of a given country. Translated into dollar values, it can cost a country's economy billions of dollars (a recent study has shown the cost of Corrosion in the USA more than US\$200b). On the other hand, similar studies and works have put the place of economic loss due to MIC between 15 to $4 \%$ of the cost of corrosion. A conservative figure of $20 \%$ would mean that in a country like the USA, the minimum direct annual cost of MIC could be in the order of US\$40b.

\section{MIC has the following characteristics}

It is an electrochemical corrosion process in nature, this means that in order to let it happen and get it going, it needs to have an anode (electron emitter), a cathode (electron receiver), an electrolyte (a medium through which ions can be transferred) and a metallic platform (through which electrons can travel). It follows from this feature that:

If there is no water or if the water has no ions (like the so-called demineralised water or what we also know as "distilled water"), electrochemical corrosion is highly unlikely to happen and so is MIC, can only be expected within the temperature range where water exists as a liquid medium to allow ion transfer, therefore in a thermal power plant it is highly likely to look for the evidence of MIC in the cold cycle (from the condenser to the boiler feed pump) than in the hot cycle (from boiler to turbine).

MIC is expected to happen only on metals because it is metals that allow electron exchange between anode and cathode. Alternatively, it is technically wrong to say "microbial corrosion of polymers" or "microbial corrosion of concrete". Instead, it is more convenient and also more correct to address it as "microbial degradation" of polymers or concrete.

MIC happens only when certain micro-and/or macro- organisms are available and active. One has to note that the mere existence of CRB/ CRA (corrosion-related bacteria/corrosion-related Archaea) does not necessarily mean that MIC could be a serious threat. It is actually if the $\mathrm{CB} / \mathrm{CRA}$ involved are active enough to create the required driving force which is necessary to advance MIC in the system of interest. The variety of micro- and macro-organisms that could be involved in any MIC process show a wide range of versatility. In other words, bacteria (and their cousins, Archaea) as well as fungi and lichen could make the conditions favourabel for inducing corrosion in the form of MIC or deterioration in the form of MID -Microbiologically
Volume 3 Issue I - 2019

\author{
Reza Javaherdashti \\ Director of Parscorrosion consultants, Monash University, \\ Australia
}

Correspondence: Reza Javaherdashti, Director of Parscorrosion consultants, Monash University, Australia, Email javaherdashti@yahoo.com

Received: February 28, 2019 | Published: March 13, 2019

influenced dterioration. An example of MIC induced by fungi could be the corrosion of aircraft body and an example of MIC by bacteria could be the extensive corrosion induced by bacteria such as, but not limited to, sulphate reducing bacteria (SRB).

The fluid by which MIC is highly likely to happen is either water or the water-phase entrained in fluids such as oil and gas. If the entrained water phase is in contact with hydro-carbon phase of fuels, this can further promote the severity of corrosion experienced by MIC.

The fluid features that are highly likely to produce an environment for initiating and sustaining MIC must have the following features:

It must be a fluid in liquid form that is either water by itself or contains water (entrained water). This fluid must also have conductivity features good enough to let ionic exchange happen. This will have two advantages from the standpoint of increasing vulnerability to corrosion and MIC: The relatively good conductivity will allow the fluid to act as an electrolyte that, when joined by suitable anode, cathode and a metallic path to allow electron flow, will create conditions favourabel for initiating and sustaining electrochemical corrosion. In addition, the watery environment is the most suitable for bacteria to allow them live in an environment most suitable for their survival and sustainability.

This fluid (water) must also have the required chemistry to support bacteria/archaea. In other words, it must have the required $\mathrm{pH}$ (not too basic and, for most of bacteria, not too acidic either except the socalled APB-acid producing bacteria such as anaerobic Clostridia and aerobic sulphur oxidisng bacteria), the required chemicals (nutrients) for growth and activity of the bacteria high total dissolved solids (TDS) content.

This fluid must also have other features as well such as suitable temperature, appropriate flow rate and characteristics. As it may be seen, the mechanical as well as chemical properties of the fluid in which MIC is likely to happen is of great importance. If the flow rate is below $1.5 \mathrm{~m} / \mathrm{s}(5 \mathrm{ft} / \mathrm{s})$, the fluid can be addressed as "stagnant". The main feature of stagnant fluid is that it allows the minimum required turbulence to destroy bacterial films that is also known as biofilms".

MIC is in fact the result of "communication" between many factors. In case the fluid that contains planktonic (freely swimming) bacteria become stagnant and motionless, its conditions along with senses of the bacteria (such as Quorum sense) will let the bacteria become 
"stuck" on the surfaces to feed on the food that are available on these surfaces. The nutrients under the effect of their weight will fall on the surfaces and as such, the planktonic bacteria would "recognize" where the food is as its concentration may have been decreased in the bulk fluid. This would drag the bacteria onto the surface and make them go through the dynamic process of biofilm formation.

MIC is a hard-to-judge phenomenon: over decades many have tried to link the failure morphology to the mechanism involved in such failures and MIC has not been an exception either. Particularly in 1980s, it was tried to show such and such features of the pitting appearance and failure morphology as a guide to let the observer know if the case was indeed related to MIC. Nowadays, however, we don't believe in such approaches. It is because by the advancement of knowledge in the field of failure analysis and investigation methods to study MIC and also other forms of corrosion, we now know that it is quite highly likely that corrosion processes other than MIC (e.g. stray current) could produce the same morphology.

Another feature that makes MIC a real challenge for both field engineers and researchers alike is that MIC has been wrongly addressed. In other words, MIC is assumed to have been caused by one or two types of bacteria, the most notorious of which being SRB. This wrong notion that has been planted in the minds of many engineers makes them believe that just because they apply some biocides (bacteria-killing chemicals) and every now and then they take a sample of the fluid for bacterial count, so the system is under control. This is certainly not true!

In addition to various types of CRB, there are also CRA. The commercial bacteria kits available in the market can only check CRB and not CRA and it is necessary to apply more sophisticated laboratory methods that are collectively referred to as molecular microbiology methods (MMM). In addition, most of these field tests can detect the planktonic CRB and not biofilm formers. This is another serious shortcoming of these so-called rapid field tests. Regarding applying biocides, it must be noted that biocide application can be expected to have a relatively significant effect only if biofilm has not been formed or its formation is still in its initial, reversible stages. When the biofilm is already formed, injection of biocide may not become that effective. For instance, if a biofilm of $1 \mathrm{~mm}$ thickness is formed on a flat substrate within three days at a temperature of $24^{\circ} \mathrm{C}$, in order to let at least $90 \%$ of any given biocide get absorbed inside such a biofilm to kill the bacteria residing there, the diffusion-based penetration process will need 12 minutes to a reach its goal. Under the very same conditions, if the biofilm gets thicker and grow to $10 \mathrm{~mm}$, the required time for the same dosage of biofilm to kill the biofilm-residing bacteria will then be 20 hours.

There are only four ways to control MIC. It must be noted that these methods are not preventive butthey are designed to control the already-happening MIC. In our professional judgment, prevention of MIC can only happen in two stages: design and operation. At design stage, the system must be designed in such a way that it will not allow sustaining of the fluid in its stagnant conditions. This would mean that in the case of a pipeline, the mere existence of bends and low-points as well as too many horizontal branches would add up more into the likelihood of fluid stagnancy. We would like to also include practices such as hydrotesting as an element of design because it could then be the hydrotest that is being done either in wrong or inadequate manner that would endanger the asset (pipeline for example) and highly vulnerable to post-hydrotesting corrosion ( that in most cases appears to be MIC). During operation, having an extensive, well-planned monitoring program in place that encompasses both MIC and nonMIC corrosion likely scenarios can significantly assist in letting an MIC preventive strategy run successfully.

\section{Returning to the MIC controlling methods, the four methods are:}

a) Mechanical-physical treatment

b) Chemical treatment

c) Electrical treatment

d) Biological treatment

Physical management of MIC can be achieved by putting a physical barrier between the vulnerable metallic substrate and the corrosive media (in case of MIC, the corrosive media would mean the electrolyte that also supports microbial growth and activity). This physical barrier is otherwise called "coating". An example of mechanical means of treatment is application of "pig"s (pipeline inspection gauge). Pigs could, in very lose terms, be divided into smart pigs and "not-smart" pigs such as cleaning pigs. All these have their own features as well as pros and cons that we may leave it to another time.

Example of chemical treatment is biocide application. Biocides are chemicals that are being applied to kill bacteria and control MIC. Biocides can be divided into oxidising and non-oxidising biocides. An example of oxidising biocides is chlorine and an example of nonoxidising biocides is Glutaraldehyde.

Cathodic protection $(\mathrm{CP})$ is an example of electrical treatment that can or can't be effective on controlling MIC depending on many factors one of which being the range within which repulsive electrostatic forces could exist between the negatively charged, CPprotected metallic surface and negatively charged bacteria. Using bacteria (or bacteria-eating organisms such as Phages) against CRB is an example of biological treatment of MIC. Contrary to the first three treatment methods, the last treatment is still not that widely used and except a few applications it is not commercially widespread, yet.

Microbiologically influenced corrosion is a very complex phenomenon. This corrosion phenomenon needs the professional attention of engineers and researchers from different backgrounds as MIC is a multi-dimensional issue per se.

The fundamental role played by the characteristics of the fluid in initiating and sustaining MIC can't be denied. These characteristics include chemical, physical, mechanical as well as biological features by which the fluid becomes the centre of attention for MIC. Realising this alone, we hope, should encourage our readers to pay much more attention to the ways by which fluids can assist in inducing corrosion and lack of mechanical integrity of the structure in question in the form of microbial corrosion.

\section{Acknowledgments}

None.

\section{Conflicts of interest}

The author declares there is no conflicts of interest. 